\title{
MAEL contributes to gastric cancer progression by promoting ILKAP degradation
}

\author{
Xing Zhang ${ }^{1, *}$, Yichong Ning ${ }^{1, *}$, Yuzhong Xiao ${ }^{2, *}$, Huaxin Duan ${ }^{3}$, Guifang Qu ${ }^{3}$, Xin \\ Liu ${ }^{1}$, Yan Du ${ }^{1}$, Dejian Jiang ${ }^{4}$ and Jianlin Zhou ${ }^{1}$ \\ ${ }^{1}$ Key Laboratory of Protein Chemistry and Developmental Biology of The Ministry of Education, College of Life Science, Hunan \\ Normal University, Changsha 410081, Hunan, China \\ ${ }^{2}$ College of Biology, Hunan University, Changsha 410082, Hunan, China \\ ${ }^{3}$ The First Affiliated Hospital, Hunan Normal University, Changsha 410005, Hunan, China \\ ${ }^{4}$ Hunan Key Laboratory of Pharmacodynamics and Safety Evaluation of New Drugs, Changsha 410331, Hunan, China \\ *These authors contributed equally to this work
}

Correspondence to: Jianlin Zhou, email: jlzhou@hunnu.edu.cn

Keywords: MAEL; gastric cancer; ILKAP; CHKI; p38 MAPK

Received: July 06, $2017 \quad$ Accepted: November 13,2017 Published: December 06, 2017

Copyright: Zhang et al. This is an open-access article distributed under the terms of the Creative Commons Attribution License 3.0 (CC BY 3.0), which permits unrestricted use, distribution, and reproduction in any medium, provided the original author and source are credited.

\section{ABSTRACT}

The cancer-testis gene MAEL is involved in the development and progression of bladder, liver and colorectal cancers. However, its role in other cancers is unclear. By systematically analyzing transcriptomics and genomics data from various cancer databases, we identified that the MAEL gene is aberrantly elevated in gastric cancer (GC) tissues and that its expression is strongly negatively correlated with DNA methylation (Pearson's correlation coefficient $=-\mathbf{0 . 6 7 5}$ ). Survival analysis revealed that MAEL expression may serve as a prognostic marker for GC patients (overall survival: hazard ratio $[H R]=1.54, p=1.2 E-4$; first progression: $H R=1.51, p=8.7 E-$ 4). In vitro and in vivo experiments demonstrated that silencing MAEL expression in the GC cell lines HGC-27 and AGS inhibits proliferation, colony formation, migration, invasion and growth of xenograft tumors, whereas MAEL overexpression exerts the opposite effects in the normal gastric cell line GES-1. Mechanistically, MAEL promotes the lysosome-dependent degradation of the protein phosphatase ILKAP, leading to increased phosphorylation of its substrates (p38, CHK1 and RSK2). Moreover, adenovirus-mediated ILKAP overexpression reversed the oncogenic effects of MAEL in vitro and in vivo. Taken together, these results indicate that MAEL exerts its oncogenic function by promoting ILKAP degradation in the GC.

\section{INTRODUCTION}

Gastric cancer (GC) is the fifth most frequently diagnosed cancer and the third leading cause of cancerrelated death worldwide [1]. The etiology of GC is very complicated; gastric carcinogenesis is related to Helicobacter pylori infection, genetic alterations, and dietary and other risk factors [2]. Even though many advances in the treatment of GC have been achieved, the 5-year survival rate of GC is still less than 30\% [2]. Tumor heterogeneity and the lack of effective therapeutic targets are the two main obstacles for precision GC medical treatments [3]. Therefore, it is urgently necessary to identify novel diagnostic, predictive, and prognostic biomarkers for targeted therapies.

Human cancers frequently exert soma-to-germline transformations by reactivating genes that are normally required for germline stemness, fitness, or longevity [4-6]. These genes, which are only expressed in germ cells and are aberrantly expressed in some cancer cells, are referred to as cancer-germline or cancer-testis genes [5]. It is commonly believed that DNA hypomethylation contributes to the abnormal activation of most cancergermline genes in somatic tissues [5]. The Maelstrom 
(MAEL) gene was first cloned in Drosophila [7], and it plays essential roles in meiosis and spermatogenesis $[8,9]$. Our previous study demonstrated that $M A E L$ is a cancer-testis gene that is only expressed in spermatocytes, round and early elongating spermatids in the testis, but is activated by demethylation in breast cancer cells [10]. Abnormal activation of MAEL is also found in liver, colon and bladder cancers and contributes to tumorigenesis, cancer cell survival, invasion and epithelial-mesenchymal transition (EMT) [11-14]. However, in contrary to what has been observed in the above cancer types, MAEL exerts a repressive effect on cell invasiveness in ovarian cancer [15]. The roles of MAEL in GC progression and development have not been investigated.

With the advances in high-throughput technologies (including microarrays and next-generation sequencing) and computational techniques, cancer research has been brought into a "big data" era. Currently, there are numerous high-throughput datasets related to cancer that are freely available in public data repositories, such as TCGA (The Cancer Genome Atlas), GEO (Gene Expression Omnibus) and EGA (European Genome-phenome Archive). These "big data" have greatly facilitated the systematic analysis of the key genes and pathways involved in tumorigenesis [16]. Here, we used cancer genomics and transcriptomics data to systematically investigate the expression and clinical significance of MAEL. We found that MAEL was significantly overexpressed in GC tissues and that high $M A E L$ mRNA levels predict poor survival in GC patients. Moreover, we demonstrated the oncogenic roles and underlying mechanisms of $M A E L$ in gastric cancer.

\section{RESULTS}

\section{MAEL mRNA is overexpressed and predicts poor survival in gastric cancers}

The cancer microarray database on the Oncomine platform was searched to analyze the differential expression of MAEL mRNA between cancerous and noncancerous tissues. The results showed that the levels of MAEL mRNA in gastric cancer (GC) (GEO: GSE27342 [17]), glioblastoma (GEO: GSE4536 [18]), invasive breast cancer (GEO: GSE9014 [19]) and lung adenocarcinoma (GEO: GSE31210 [20]) were significantly higher than their corresponding normal tissues (fold change $\geq 2$, $p$-value $<0.01$ ) (Figure $1 \mathrm{~A}$ and Supplementary Figure $1 \mathrm{~A}-1 \mathrm{C}$ ) and that $M A E L$ ranked in the top $6 \%, 10 \%, 16 \%$ and $20 \%$ of overexpressed genes in gastric, brain, breast and lung cancers, respectively.

The impact of $M A E L$ expression on survival was investigated using Kaplan-Meier Plotter, which includes the gene expression data and survival information from the breast, ovarian, lung and gastric cancer patients [21]. The results revealed that higher $M A E L$ mRNA expression was significantly associated with a shorter overall survival (OS) (hazard ratio $[\mathrm{HR}]=1.54,95 \%$ confidence interval [CI]: $1.23-1.92$, logrank $p$-value $[p]=1.2 \mathrm{E}-4)$ and first progression survival $(\mathrm{FP})(\mathrm{HR}=1.51, \mathrm{CI}: 1.18-1.93$, $p=8.7 \mathrm{E}-4$ ) in gastric cancer patients (Figure $1 \mathrm{~B}$ and $1 \mathrm{C}$ ). However, there was no significant correlation between $M A E L$ expression and the OS of patients with breast $(\mathrm{HR}=0.89$, C CI: $0.69-1.27, p=0.53)$, lung (HR = 1.07, CI: $0.9-1.26, p=0.45)$ or ovarian $(\mathrm{HR}=1.02, \mathrm{CI}: 0.82-1.28$, $p=0.85$ ) cancer (Supplementary Figure 1D-1F). These results indicated that $M A E L$ expression in GC tissues is an unfavorable prognostic marker for GC patients.

According to the above results of meta-analysis, we were interested in the roles of MAEL gene in the GC. Therefore, we verified $M A E L$ mRNA expression using real time PCR and MAEL protein level using Western blotting in 4 pairs of gastric tumor and adjacent normal tissues and 8 gastric cell lines. In consistent with the result of Oncomine analysis (Figure 1A), the average level of $M A E L$ mRNA in gastric tumor tissues was significantly higher than that in the adjacent noncancerous tissues (Figure 1D); MAEL protein levels were also higher in the cancer tissues compared to the noncancerous tissues in 3 out of 4 of the tissue pairs investigated (Figure 1F). In gastric cell lines, the cancer cells HGC-27, AGS and KATOIII displayed significantly higher levels of both mRNA and protein than the normal cells GES-1 and RGM1 (Figure 1E and 1G).

\section{$M A E L$ expression is induced by hypomethylation in gastric cancers}

To investigate whether $M A E L$ expression was regulated by gene amplification or DNA methylation, we analyzed the correlation between DNA variation and mRNA expression using cBioPortal based on the Stomach Adenocarcinoma data (TCGA, Provisional). The rates of DNA mutation and copy number variation are $2.4 \%$ and $1.8 \%$, respectively, among the 478 gastric adenocarcinoma tissue cases. The MAEL mRNA levels are weakly correlated with the $M A E L$ gene copy number (Pearson's correlation coefficient $(r)=0.119$ ) and strongly negatively correlated with methylation of $\mathrm{CpG}$ island in the MAEL gene promoter (Pearson's $r=-0.675$ ) (Figure 2A). We verified the $\mathrm{CpG}$ island methylation of $M A E L$ gene promoter among 4 pairs of gastric tumor and adjacent normal tissues and 8 gastric cell lines using pyrosequencing (Figure 2B and Supplementary Figure 2). Pearson's correlation analysis was used to analyze the correlation between $\mathrm{CpG}$ methylation level and mRNA expression, and revealed a significantly negative relationship (Pearson's $r=-0.787$ ) (Figure 2B). This result is consistent with that of cBioPortal analysis. Additionally, we treated the cell lines (BGC-823, RGM1 and GES-1) with the demethylating agent 5-Aza-2-Deoxycytidine 
(5-AZA), which significantly upregulated levels of both MAEL mRNA and protein (Figure 2C and 2D), suggesting that DNA hypomethylation in the $M A E L$ gene promoter possibly contributes to its overexpression in GC cells.

\section{$M A E L$ knockdown inhibits gastric cancer progression}

To investigate the role of $M A E L$ in GC cells, we used a lentivirus-delivered shRNA system to generate stable GC cell lines (HGC-27 and AGS) that express scrambled or MAEL shRNA (Figure 3A). MTT assay revealed that $M A E L$ knockdown inhibited cell proliferation in both AGS and HGC-27 cells (Figure 3B). In a colony formation assay, fewer colonies were observed in the stable AGS and HGC-27 cells, whose MAEL expression was inhibited compared with mock cells or cells infected with the empty lentivirus vector (Figure 3C). The wound-healing assay showed that the $M A E L$-silenced cells demonstrated slower closure of the scratched wound compared with the control cells, indicating that MAEL depletion inhibits cell migration (Figure 3D). In the transwell invasion assay, we observed that the number of invaded cells decreased when $M A E L$ expression was suppressed by shRNAs (Figure 3E). Silencing MAEL expression in SUN-1 cells also inhibited cell growth, colony formation, wound healing and invasion (Supplementary Figure 3). These results demonstrated that $M A E L$ participates in $\mathrm{GC}$ cell proliferation, migration and

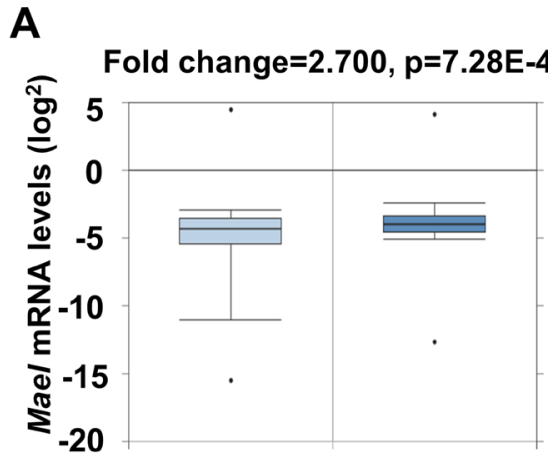

Normal $(n=80)$ Tumor $(n=80)$

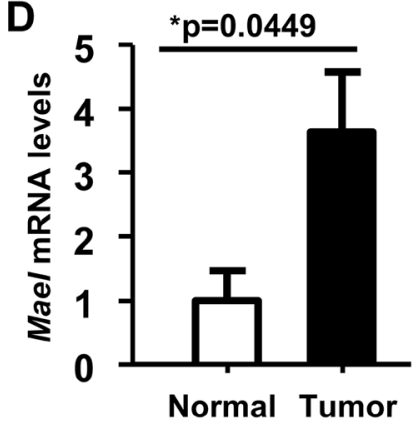

$\mathbf{F}$

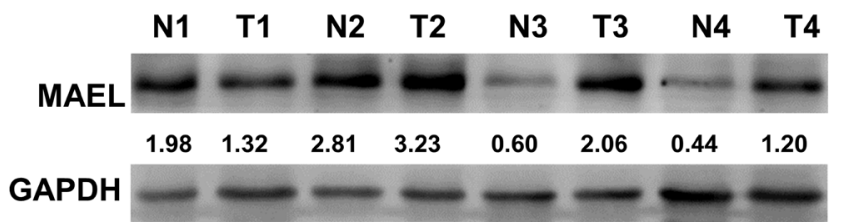

B

$E$
$H R=1.54$ (1.23-1.92), $p=1.2 E-4$
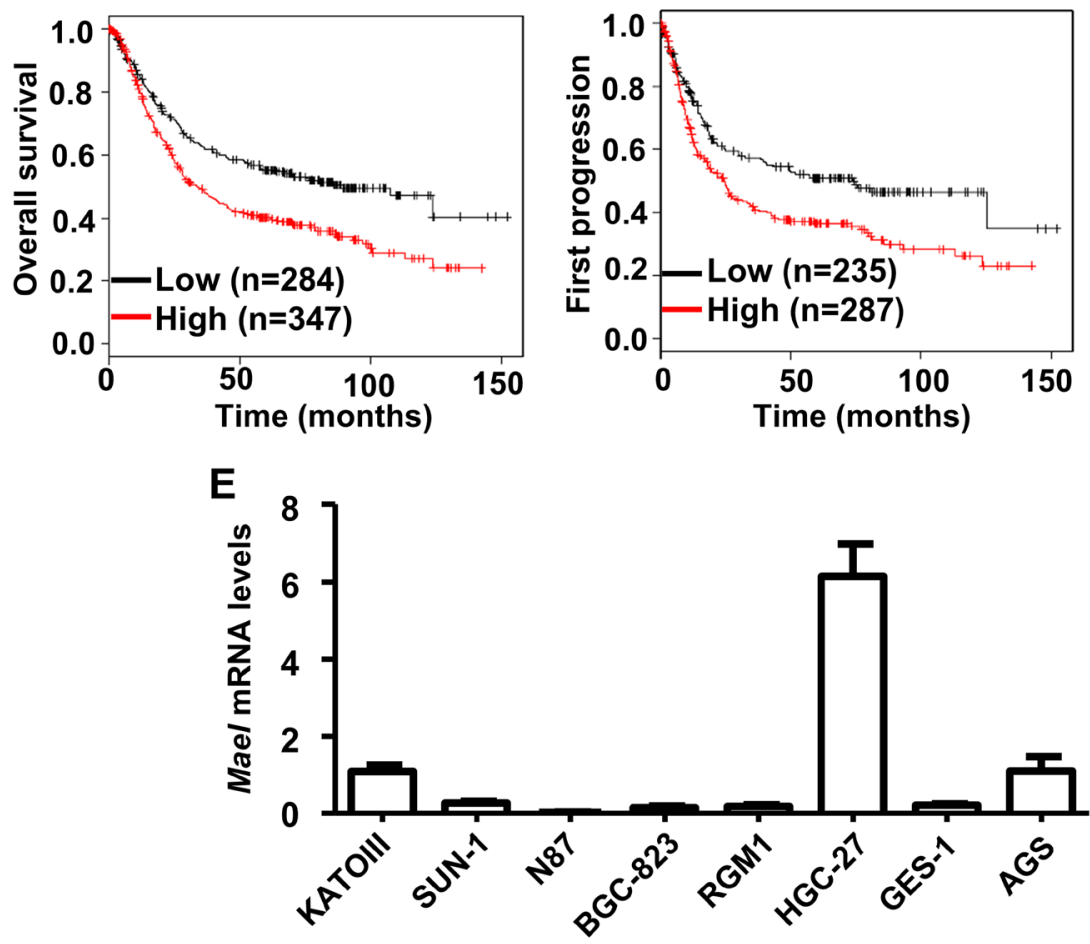

G

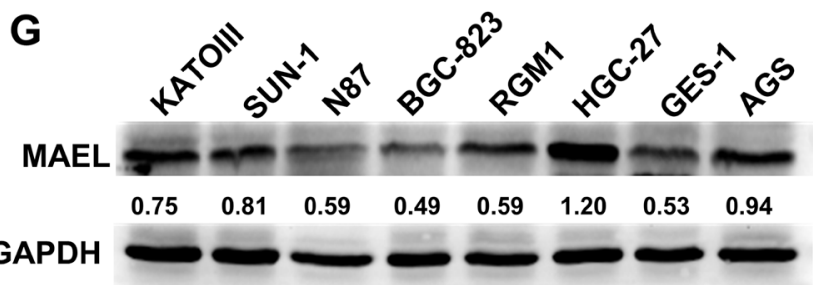

Figure 1: Expression and prognostic significance of $\boldsymbol{M A E L}$ gene in gastric cancer. (A) Differential expression of $M A E L$ mRNA between gastric normal and tumor tissues were analyzed by Onocomine based on the NCBI GEO dataset GSE27342. (B, C) Correlation of MAEL mRNA and overall/first-progression survival in gastric cancer was calculated using Kaplan Meier plotter based on the NCBI GEO datasets (GSE14210, GSE15459, GSE22377, GSE29272, GSE51105, and GSE62254). HR: hazard ratio, n: sample size. (D, E) The MAEL mRNA expression in the paired gastric tumor and adjacent normal tissues (D) and cell lines $(\mathbf{E})$ were detected by realtime PCR. The MAEL mRNA expression levels were normalized using GAPDH control, and expressed as the mean \pm standard deviation (S.D) of three replicates. $(\mathbf{F}, \mathbf{G})$ The MAEL protein levels in the paired gastric tumor and adjacent normal tissues $(\mathbf{F})$ and cell lines $(\mathbf{G})$ were detected by Western blotting. Staining intensity was quantitated by Image J software. The number below the blots indicates relative band intensity of MAEL protein normalized against that of GAPDH. N: normal adjacent noncancerous tissue, T: tumor tissue. 
invasion. Additionally, we further investigated the in vivo effects of $M A E L$ on tumor growth using a subcutaneous xenograft tumor mouse model. As shown in Figure $4 \mathrm{~A}-4 \mathrm{C}$, the tumors derived from $M A E L$-depleted HGC27 cells grew significantly slower than tumors derived from control cells (Figure 4A); at the termination of the experiment, the average weights of the tumors derived from $M A E L$-depleted HGC-27 cells was much less than the controls (Figure 4B and 4C). Conversely, $M A E L$ overexpression in GES-1 cells promotes the formation and growth of xenograft tumors (Figure 4D-4F).

\section{MAEL interacts with ILKAP and promotes its lysosome-dependent degradation}

In our previous study, ILKAP was identified to be a component of the MAEL protein complex [22]. To confirm the protein interaction between MAEL and ILKAP, we performed co-immunoprecipitation (co-IP). We cotransfected a Myc-tagged MAEL expression plasmid and an HA-tagged ILKAP expression plasmid into HEK293 cells. At $24 \mathrm{~h}$ post-transfection, cell lysates were prepared and immunoprecipitated with anti-HA antibody or preimmune IgG. Western blot assay showed that MAEL could be detected in the immune complex precipitated with antiHA antibody, but not in complexes precipitated with IgG (Figure 5A). Moreover, co-IP experiment of endogenous MAEL and ILKAP proteins in AGS cells showed that endogenous MAEL protein could be precipitated efficiently by anti-ILKAP antibody, but not by IgG (Figure 5B). These results suggested that MAEL can interact with ILKAP.

To map the interaction site, we performed coIP experiments using the HEK293 cells co-transfected with HA-ILKAP and Myc-tagged deletion mutants of MAEL. The results (Figure 5C) showed that deletion of the MAEL domain of MAEL protein disrupted the interaction between MAEL and ILKAP, suggesting that the MAEL domain is responsible for interaction with ILKAP. Interestingly, we found that ILKAP protein level in the cells co-transfected with HA-ILKAP and Myc-
A

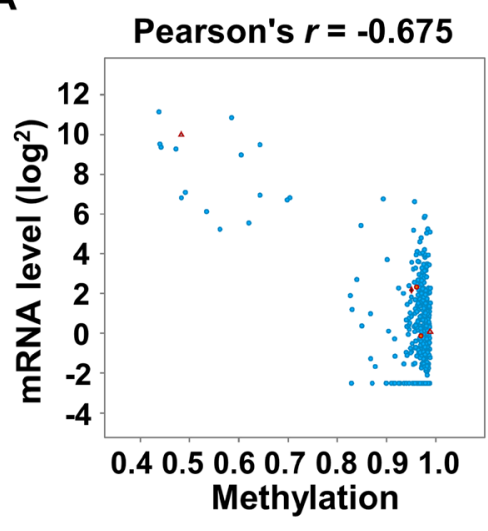

Pearson's $r=0.119$

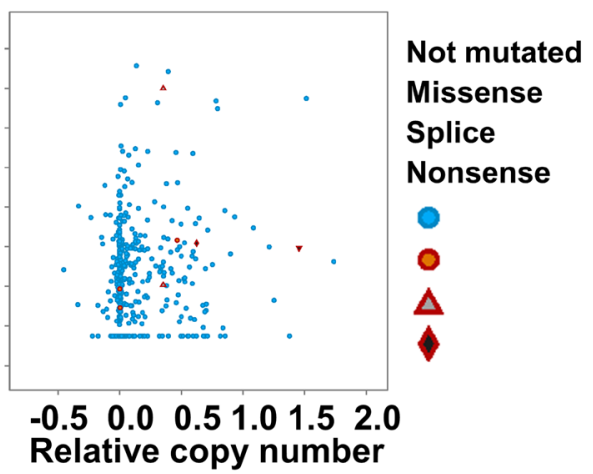

B

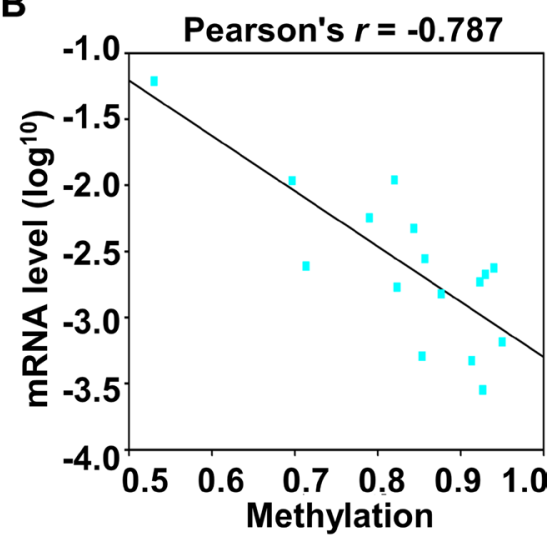

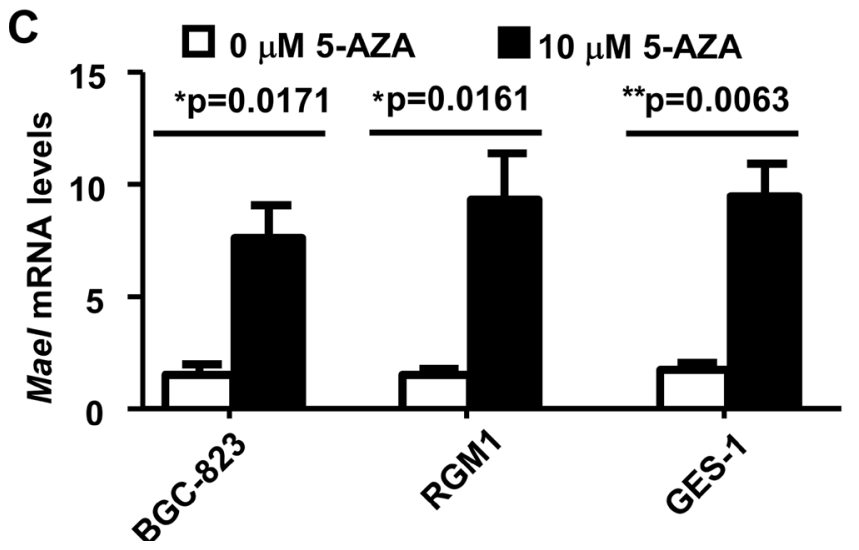

D

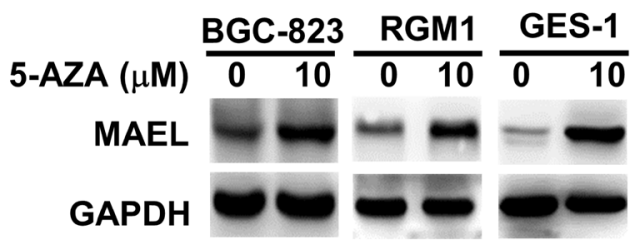

Figure 2: Expression of $M A E L$ gene is regulated by promoter methylation in gastric cancer. (A) Correlation between MAEL expression and DNA methylation/gene copy number were analyzed by cBioPortal based on the TCGA data derived from 478 gastric adenocarcinoma patients. (B) Correlation between MAEL expression and DNA methylation in the 4 pairs of gastric tumor and adjacent normal tissues and 8 gastric cell lines. The DNA methylation of MAEL gene promoter was detected by pyrosequencing assay, and the correlation between methylation and mRNA expression (in Figure 1D and 1E) was calculated and plotted by SigmaPlot (Systat Software, San Jose, CA). (C, D) Effects of treatment with 5-AZA on the levels of MAEL mRNA and protein. Cells were treated with or without 5-AZA for $72 \mathrm{~h}$, and then harvested for isolation of mRNA and protein. The levels of MAEL mRNA and protein were detected by realtime PCR and Western blotting, respectively. 
A

Mock NC shMAEL

Mock NC ShMAEL

- MAEL $\mid \oint$
B

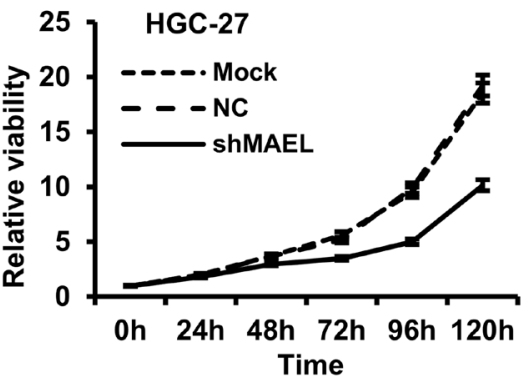

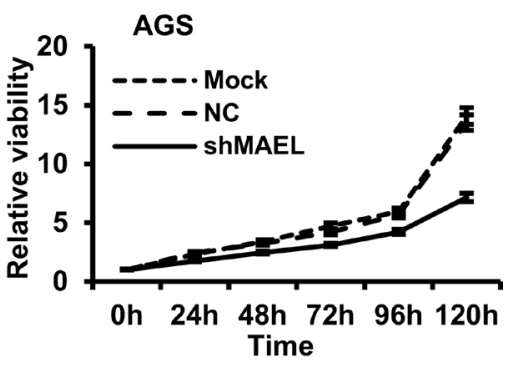

C
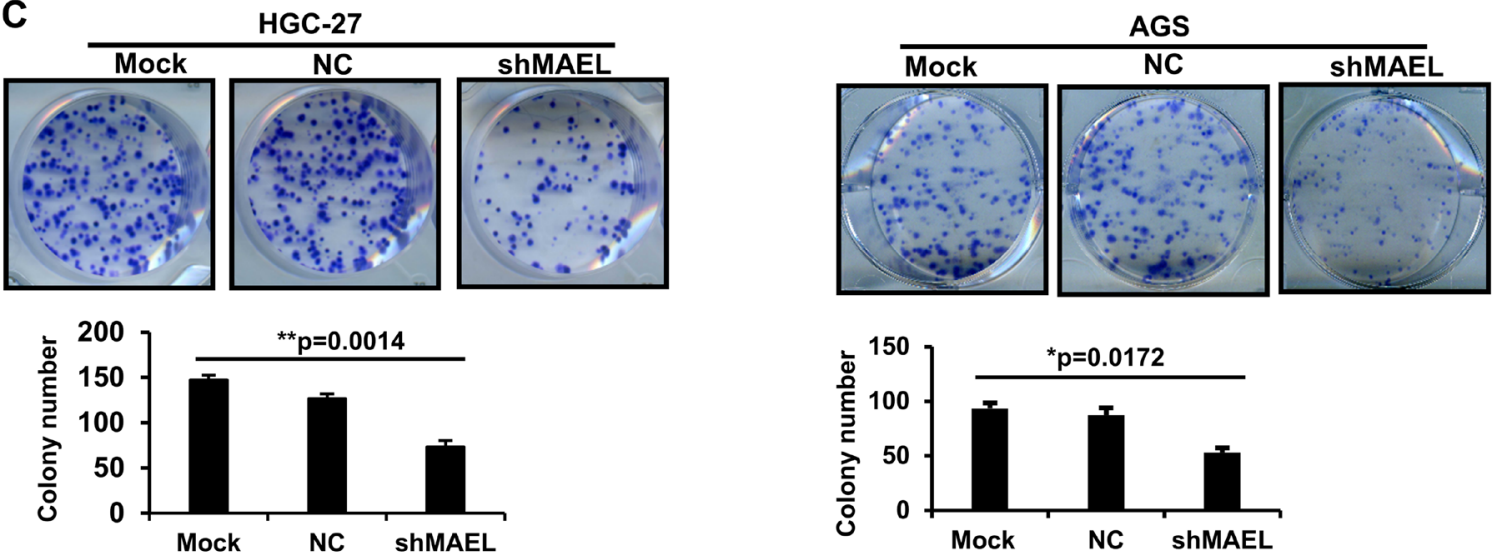

D
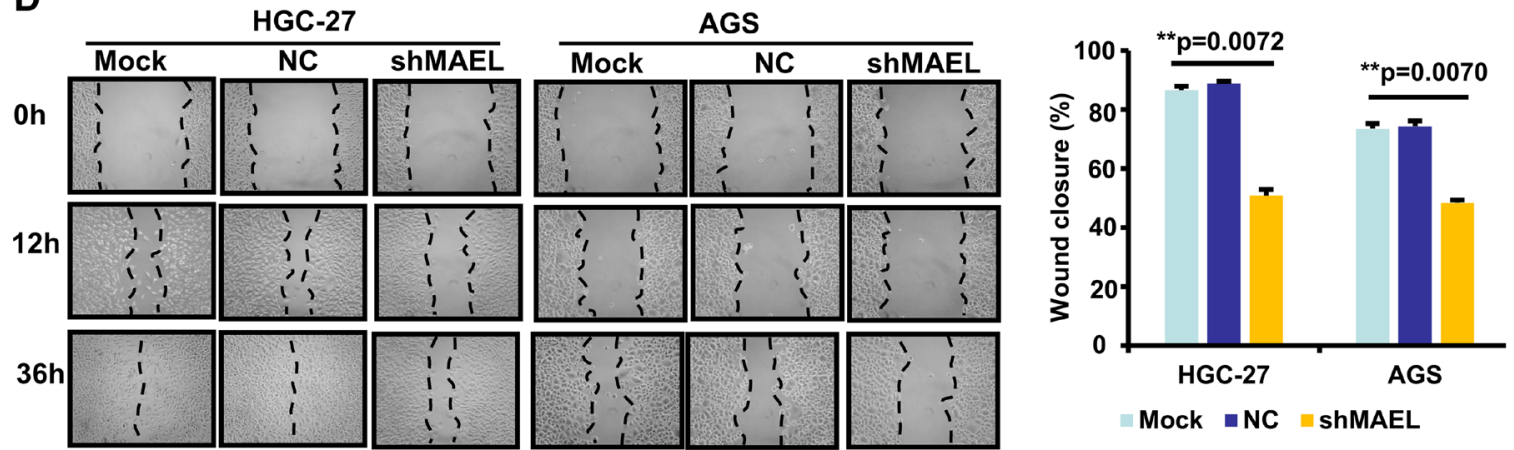

E
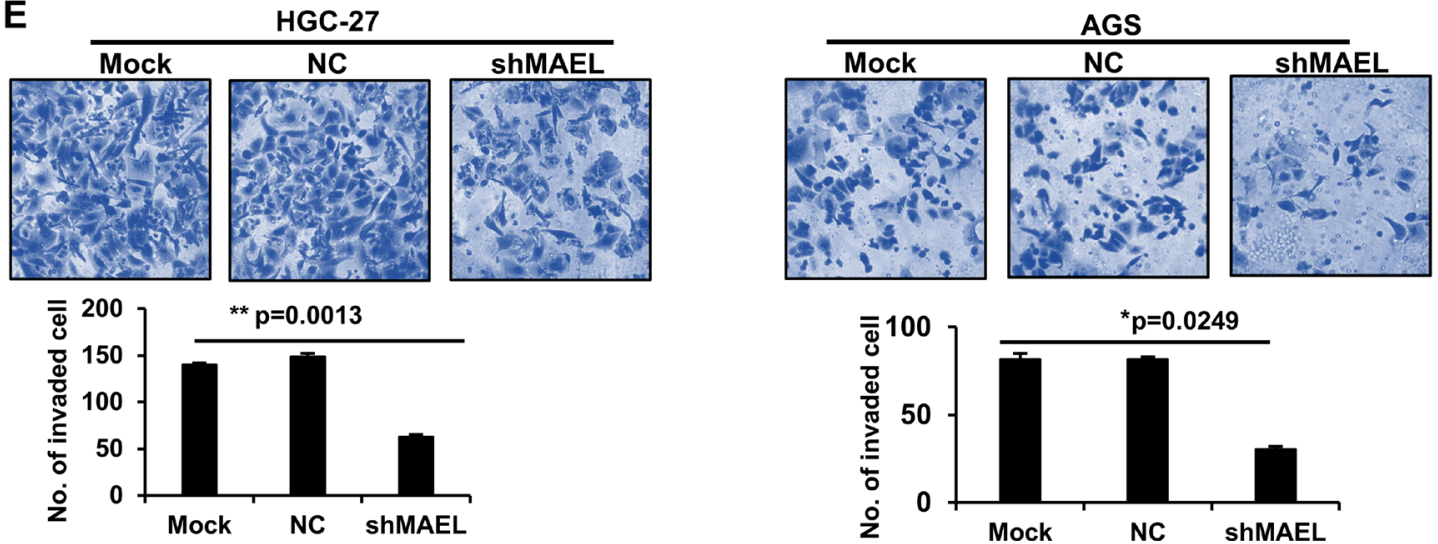

Figure 3: MAEL knockdown inhibits proliferation, migration and invasion in HGC-27 and AGS GC cells. (A) MAEL protein levels in the untreated GC cells (mock) and the GC cells with stably expressing MAEL shRNA (shMAEL) or scrambled shRNA (NC). (B) The cell growth was determined by MTT assay. (C) Representative images of colony (upper panel) and the colony numbers (lower panel). (D) Representative images (left panel) of wound area at the indicated time and percentage of wound closure at $36 \mathrm{~h}$ after scratching (right panel). (E) Representative images (upper panel) and statistical analysis (lower panel) of invaded cells in the Transwell invasion assay. All the values were presented as means \pm S.D for at least three independent in vitro experiments. Differences between two groups were analyzed by student $t$-test; ${ }^{*} p$-value $\leq 0.05$ and ${ }^{* *} p$-value $\leq 0.01$. 
$M A E L$ expression plasmid was much lower than that in the cells transfected with HA-ILKAP and empty vector, suggesting that MAEL decreases ILKAP protein levels. Then, we transfected a specific amount of the HA-ILKAP plasmid and increasing amounts of wild-type or MAEL domain deleted MAEL expression plasmid into HEK293 cells, and we found that increasing wild-type $M A E L$ expression significantly decreased ILKAP protein levels, but MAEL domain deleted mutant did not affect ILAKP protein levels, indicating that MAEL mediated ILKAP degradation is protein-protein interaction dependent (Figure 5D). Thus, the effect of MAEL on ILKAP protein turnover was further investigated. The results revealed that ILKAP degradation occurred much more rapidly in the cells transfected with the Myc-MAEL plasmid than the empty vector (Figure 5E). To assess which pathway may affect ILKAP stability, we treated the cells with proteasome, lysosome or autophagy inhibitor. We observed that MAEL-induced ILKAP degradation could be rescued by the lysosome inhibitors Leupeptin, Chloroquine and $\mathrm{NH} 4 \mathrm{Cl}$ and the autophagy inhibitor 3-MA, but not by the proteasome inhibitor MG132, indicating that ILKAP is degraded through an autophagy/lysosome-mediated pathway (Figure 5F).

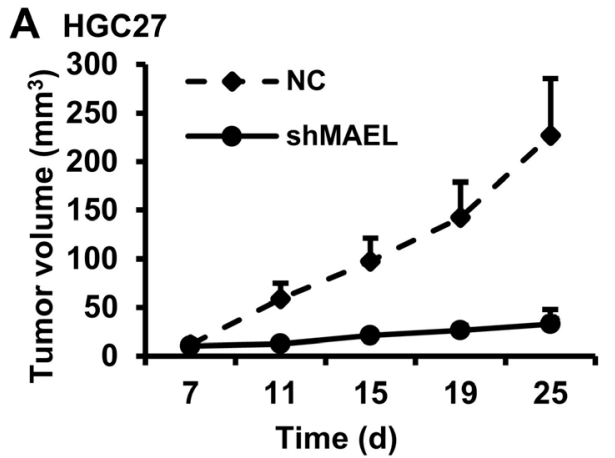

C ${ }_{\text {HGC27 }}$

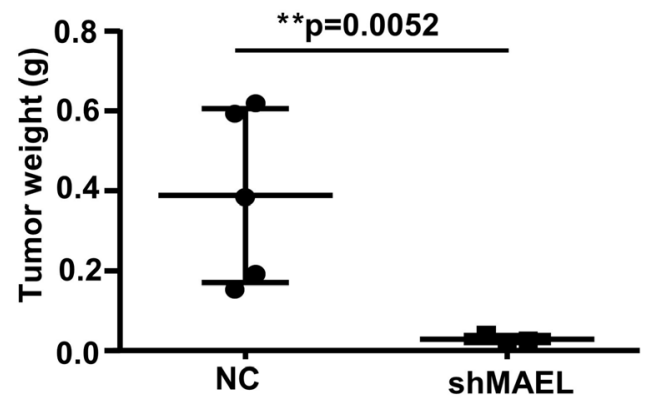

$\mathbf{E}$

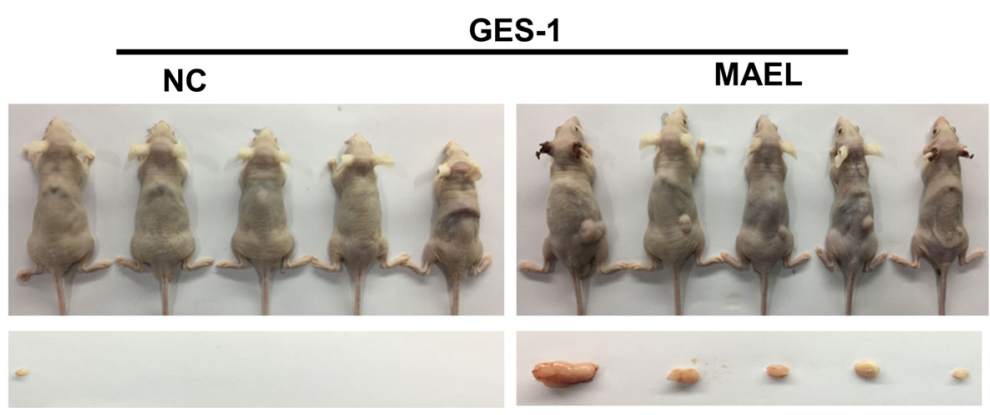

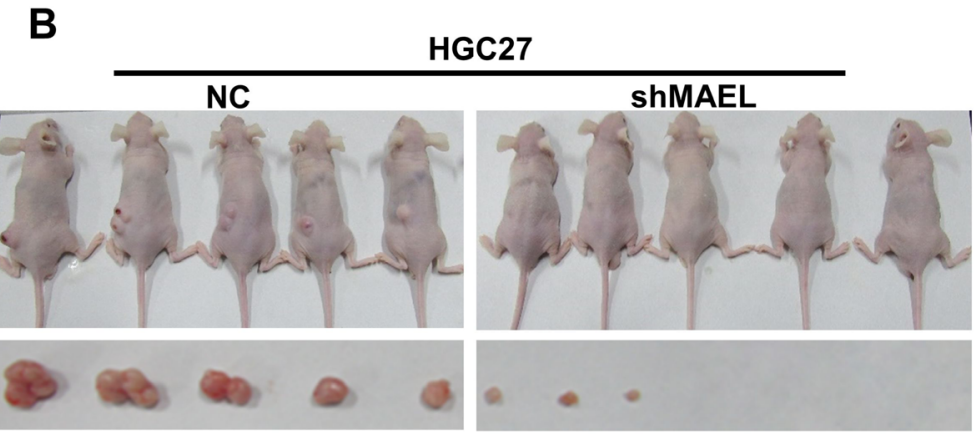

D GES-1
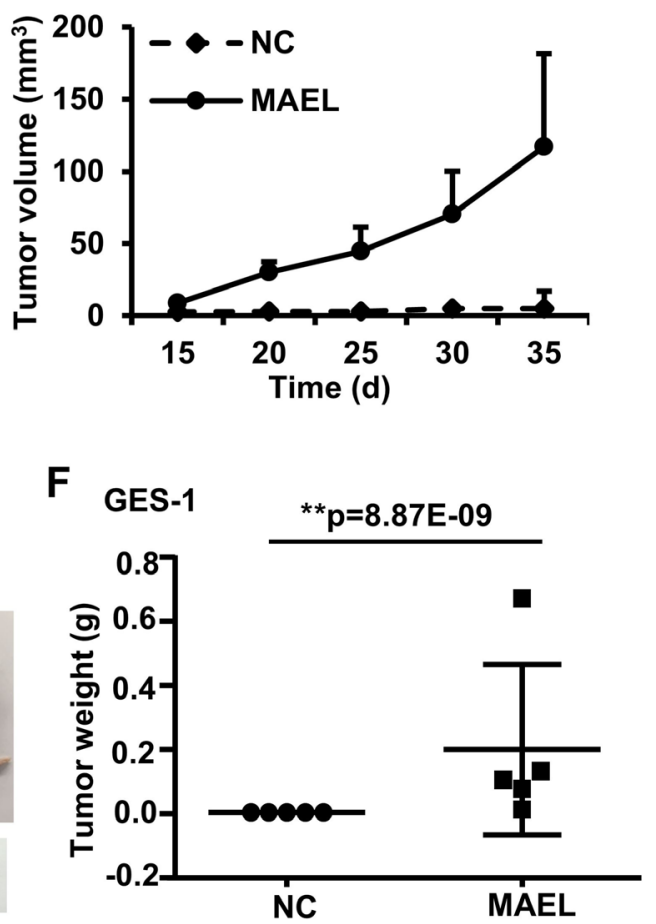

Figure 4: The influence of $M A E L$ on the growth of xenograft tumors. (A-C) $M A E L$ knockdown in HGC-27 cell inhibits the growth of xenograft tumors. BALB/c nude mice were injected with $5 \times 10^{6} \mathrm{HGC}-27$ cells expressing scrambled shRNA (NC) or MAEL shRNA (shMAEL). (A) Dynamic volume (mean \pm S.D, $n=5$ ) of xenograft tumors at different time after injection. (B) Images of the nude mice and their xenograft tumors at $30 \mathrm{~d}$ after injection. (C) Weight (mean $\pm \mathrm{SD}, n=5$ ) of xenograft tumors at $30 \mathrm{~d}$ after injection. (D-F) MAEL overexpression in GES-1 cell promotes the growth of xenograft tumors; BALB/c nude mice were injected with $1 \times 10^{7}$ GES- 1 cells expressing empty vector (NC) or MAEL cDNA. (D) Dynamic volume (mean $\pm \mathrm{SD}, n=5$ ) of xenograft tumors at different time after injection. (E) Images of the nude mice and their xenograft tumors at $40 \mathrm{~d}$ after injection. (F) Weight (mean $\pm \mathrm{SD}, n=5$ ) of xenograft tumors at $40 \mathrm{~d}$ after injection. Differences between two groups were analyzed by student $t$-test, ${ }^{*} p$-value $\leq 0.05$ and ${ }^{* *} p$-value $\leq 0.01$. 


\section{MAEL stimulates p38 MAPK, CHK1 and RSK2 phosphorylation by inhibiting ILKAP}

The above results showed that MAEL promoted ILKAP degradation. ILKAP is a member of the serine/ threonine phosphatase 2C (PP2C) family [23]. Therefore, we wanted to know whether MAEL affects the phosphorylation of ILKAP substrates. p38 MAPK (p38), CHK1 and RSK2 have been identified as potential ILKAP substrates [24]. First, we transfected HA-ILKAP plasmid
A

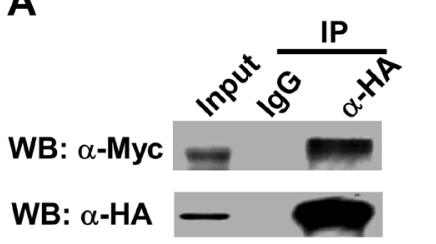

B

WB: $\alpha-M A E L$

WB: $\alpha$-ILKAP

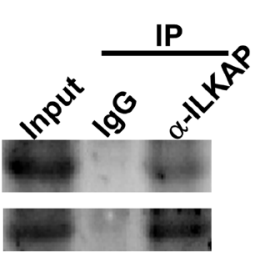

C
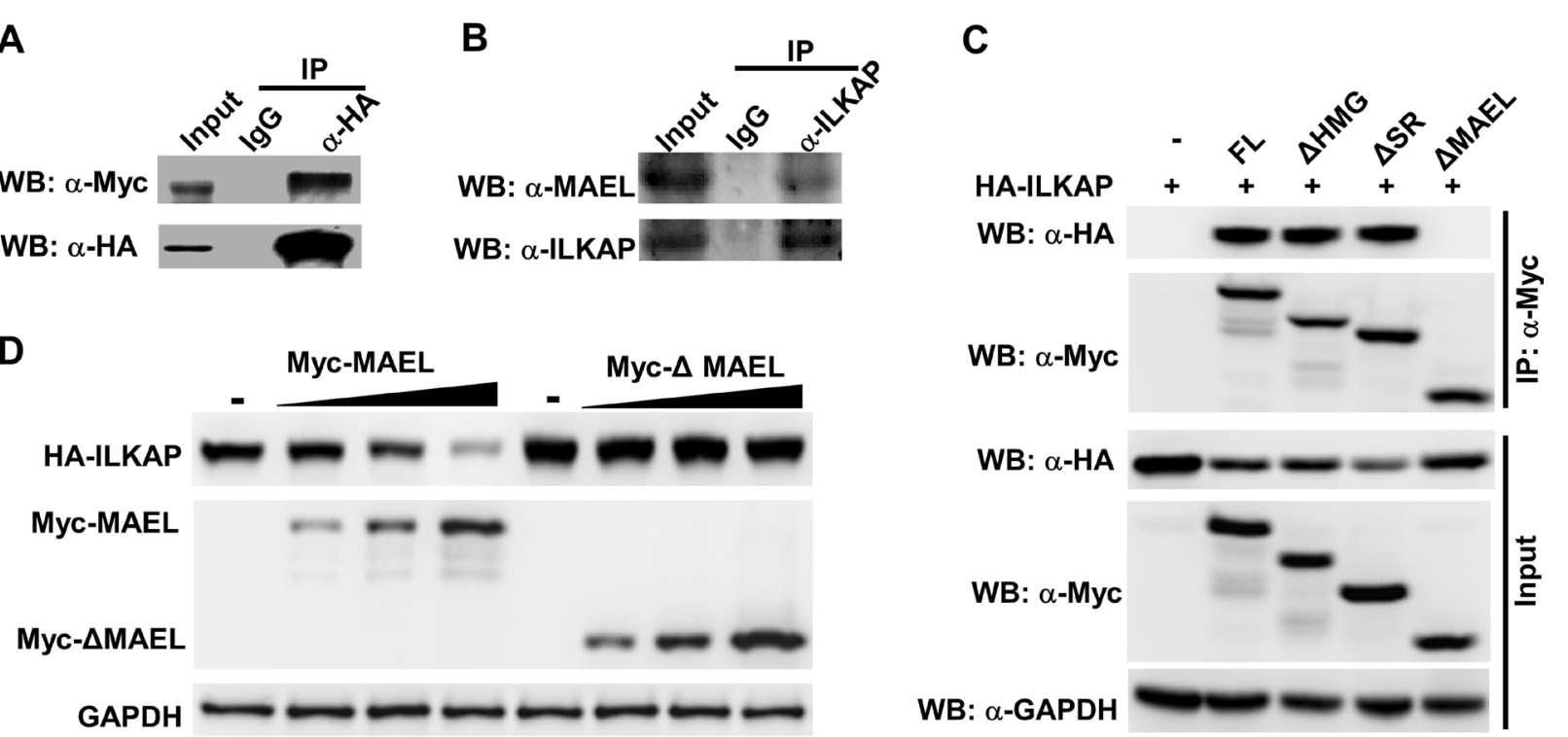

WB: $\alpha$-GAPDH

E

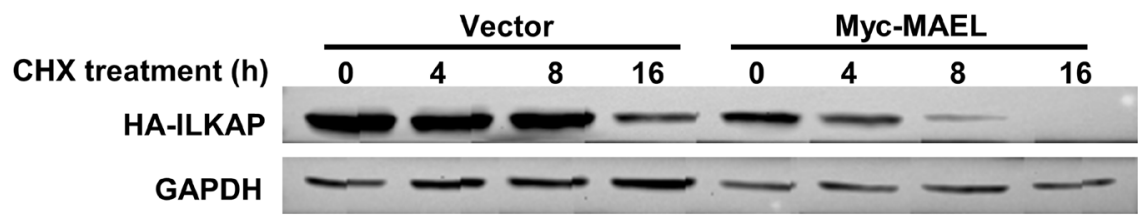

$\mathbf{F}$

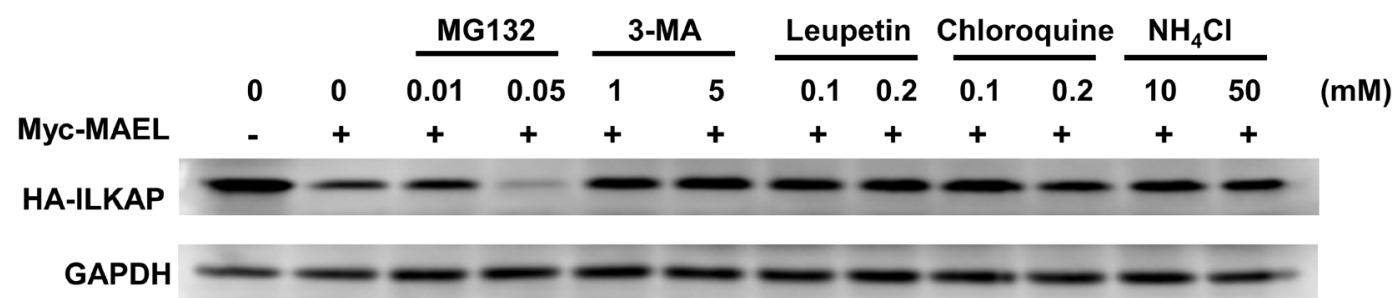

Figure 5: MAEL protein interacts with ILKAP protein and promotes its lysosomal-dependent degradation. (A) Co-IP assay in HEK293 cells transiently transfected with HA-ILKAP and Myc-MAEL. Cell lysates (800 $\mu \mathrm{g})$ were precipitated with anti-HA antibody or control IgG, and the immune complexes were subjected to Western blotting with anti-HA or anti-Myc antibody. Input is equivalent to $10 \%$ of the lysate used for the co-IP. (B) Co-IP of endogenous MAEL and ILKAP proteins in AGS cells. Cell lysates ( $800 \mu \mathrm{g})$ from AGS were precipitated with anti-ILKAP antibody or control IgG, and the immune complexes were subjected to Western blotting with anti-MAEL and anti-ILKAP antibody. Input is equivalent to $10 \%$ of the lysate used for the co-IP. (C). Co-IP assay in HEK293 cells transiently transfected with HA-ILKAP and Myc-tagged deletion mutant of MAEL gene. Cell lysates ( $800 \mu \mathrm{g})$ were precipitated with antiMyc antibody, and the immune complexes were subjected to Western blotting with anti-HA or anti-Myc antibody. Input is equivalent to $10 \%$ of the lysate used for the co-IP. FL: full length MAEL, $\triangle \mathrm{HMG}$ : HMG domain (aa 5-72) deletion, $\triangle$ SR: Ser-rich domain (aa 353-434) deletion, $\triangle$ MAEL: MAEL domain (aa 130-326) deletion. (D) MAEL overexpression decreases the ILKAP protein levels. HEK293 cells were co-transfected with a constant amount of HA-ILKAP plasmid and increasing amount of Myc-MAEL or Myc- $\triangle$ MAEL plasmid, cell lysates were prepared and subjected to Western blotting using anti-HA and anti-Myc antibodies at $24 \mathrm{~h}$ post-transfection. (E) MAEL overexpression decreases ILKAP stability. The HEK293 cells were co-transfected with HA-ILKAP and Myc-MAEL or empty vector and incubated with cycloheximide (CHX, $50 \mathrm{\mu g} / \mathrm{ml}$ ) for different lengths of time, the cell lysates were subjected to Western blotting using antiHA and anti-Myc antibodies. (F) Treatment with lysosome/autophagy inhibitor rescues ILKAP levels. The HEK293 cells were transfected with HA-ILKAP and Myc-MAEL and treated with CHX plus proteasome/lysosome inhibitors for $4 \mathrm{~h}$, HA-ILKAP protein levels were detected by Western blotting using anti-HA antibody. 
with or without Myc-MAEL into HEK293 cells and found that $M A E L$ overexpression augmented phosphorylated $\mathrm{p} 38$ (at Thr180/Thr182) levels, whereas ILKAP overexpression inhibited p38 phosphorylation (Figure 6A). Further study showed that MAEL depletion increased ILKAP protein levels and decreased p38, CHK1 (at Ser345) and RSK2 (at Ser227) phosphorylation in HGC-27 cells (Figure 6B), while MAEL overexpression exerted the opposite effects on ILKAP and its substrates in GES-1 cells (Figure 6C). In the gastric tissues and cell lines, ILKAP appeared to have an opposite expression pattern to MAEL and the phosphorylated CHK1, RSK2 and p38 (Supplementary Figure 4A and 4B). Moreover, in $M A E L$-overexpressing GES-1 cells, the increased phosphorylation of p38, CHK1 and RSK2 by MAEL overexpression was almost completely abrogated by adenovirus-mediated ILKAP overexpression (Figure 6D). Taken together, these results indicated that ectopic expression of MAEL contributes to increased p38, CHK1 and RSK2 phosphorylation by inhibiting ILKAP in gastric cancer cells.

ILKAP has been proven to selectively inhibit ILK-mediated GSK3 $\beta$ phosphorylation on Ser9, though it does not affect AKT phosphorylation [25, 26]. However, we did not observe significant effects of $M A E L$ expression on either AKT (at Ser473) or GSK3 $\beta$ (at Ser9) phosphorylation (Figure 6B and 6C).

\section{$I L K A P$ suppresses the oncogenic role of $M A E L$}

ILKAP has been found to play tumor suppressive roles by catalyzing the dephosphorylation of some oncogenic kinases, such as RSK2 [27]. To confirm whether the oncogenic role of $M A E L$ is mediated by decreasing ILKAP protein levels, we investigated the influence of ILKAP rescue on the oncogenic roles of $M A E L$. GES-1 cells stably expressing $M A E L$ were generated via a lentivirus-mediated expression system and then infected with adenoviruses expressing ILKAP or GFP. As shown in Figure 7, MAEL overexpression promoted cell proliferation, colony formation, cell migration and invasion, while adenovirus-mediated ILKAP overexpression reversed the oncogenic transformation induced by $M A E L$ overexpression in GES-1 cells. $M A E L$ has been found to be involved in the process of epithelial-mesenchymal transition (EMT) [11, 12]. We investigated the effects of $M A E L$ on an epithelial marker E-cadherin and a mesenchymal marker N-cadherin levels, and found that $M A E L$ overexpression suppressed the E-cadherin expression and induced $\mathrm{N}$-cadherin expression, whereas ILKAP overexpression abrogated the effects of $M A E L$ on expression of both E-cadherin and N-cadherin (Supplementary Figure 4C). We further confirmed influence of ILKAP rescue on the oncogenic roles of MAEL in GC cell line BGC-823 (Supplementary Figure 5). To evaluate the in vivo effects of ILKAP and $M A E L$ on tumor growth, a xenograft tumor model was generated. When the tumor volumes reached 50-100 $\mathrm{mm}^{3}$, adenoviruses expressing ILKAP or GFP were injected into the tumors. Based on our results, injection of adenoviruses expressing ILKAP significantly suppressed the growth of xenograft tumors derived from HGC-27 cells (Supplementary Figure 6). Taken together, these data demonstrated that ILKAP suppresses the oncogenic roles of MAEL.

\section{DISCUSSION}

The cancer-testis gene $M A E L$ has been found to exert oncogenic effects in liver, bladder and colorectal cancers [11-13], but anti-oncogenic effects in ovarian cancer [15]. In this study, we found that MAEL mRNA was expressed at higher levels in gastric cancer, glioblastoma, invasive breast cancer and lung adenocarcinoma than in their corresponding normal tissues (fold change $\geq 2$, $p$-value $<0.05)$ by online analysis of transcriptomic datasets from cancer patients on the Oncomine platform. Kaplan-Meier Plotter analysis revealed that high $M A E L$ mRNA levels predicted poor survival in GC patients but was not associated with overall survival in breast, lung and ovarian cancers. Furthermore, silencing $M A E L$ expression in GC cells suppressed cell proliferation, colony formation, migration, invasion and the growth of xenograft tumors, while its overexpression exerts the opposite effects in normal gastric epithelial cells. These findings strongly suggest that $M A E L$ plays an oncogenic role in GC progression.

Most cancer-testis genes are activated by DNA hypomethylation in cancer cells [5], and MAEL expression has been reported to be regulated by DNA methylation in breast and colorectal cancer $[10,28]$. According to our analysis of the transcriptomics and epigenomics data from TCGA database using the cBioPortal tool, $M A E L$ expression is strongly negatively correlated with DNA methylation status (Pearson's $r=-0.675$ ) in GCs. We verified the $\mathrm{CpG}$ island methylation of $M A E L$ gene promoter among gastric patient specimens and cell lines using pyrosequencing, the result was consistent with that of cBioPortal analysis. Moreover, treatment with demethylating agents enhanced $M A E L$ expression, suggesting that high $M A E L$ mRNA level may result from DNA hypomethylation in gastric cancer.

In consistent with the studies in liver, bladder and colorectal cancers [11-13], we demonstrated that MAEL promote tumor growth and progression in GCs. However, the underlying mechanism is not exactly the same in different types of cancer. In liver cancer, MAEL play roles by activation of Akt/GSK-3b/Snail signaling [11]; in colorectal cancer, MAEL and Snail synergistically inhibits the transcription of E-cadherin [12]; MAEL enhances the binding of DNMT3B to the promoter of MTSS1, and thereby epigenetically suppressing the MTSS1 transcription in bladder cancer [13]. Here, we demonstrated 
A

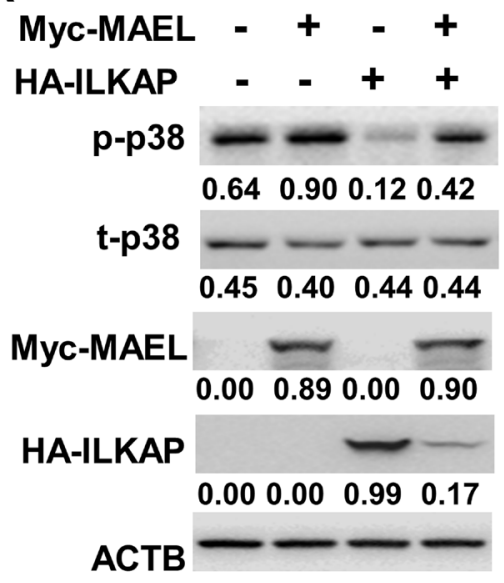

B

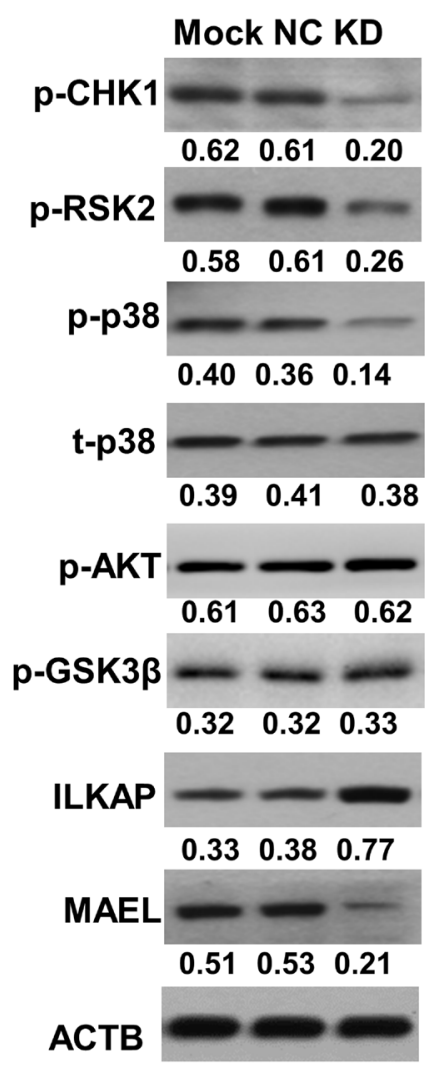

C
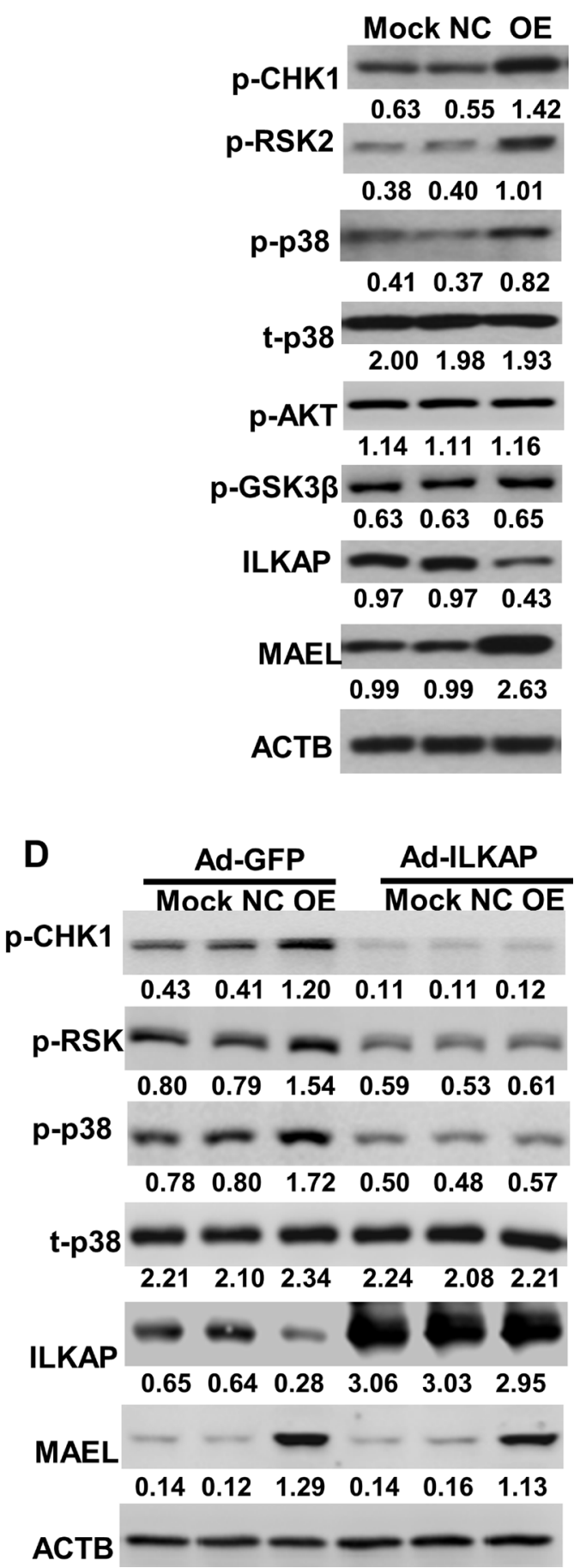

Figure 6: MAEL suppresses ILKAP-mediated dephosphorylation. (A) MAEL overexpression increases while ILKAP overexpression decreases p38 phosphorylation in HEK293 cells. HEK293 cells were transiently transfected with Myc-MAEL or/and HAILKAP expression plasmid. At $24 \mathrm{~h}$ post-transfection, the phosphorylation of p38 was detected using Western blot with anti-phospho-p38 (T180/T182) antibody. (B) Western blot shows that MAEL knockdown increases ILKAP protein levels but suppresses the phosphorylation of its substrates in HGC-27 cells. Mock: uninfected HGC-27 cells, NC: HGC-27 cells stably expressing scrambled shRNA, KD: HGC-27 cells stably expressing MAEL shRNA. (C) MAEL overexpression decreases ILKAP protein levels but increases the phosphorylation of its substrates in GES-1 cells. Mock: GES-1 cells, NC: GES-1 cells stably expressing empty vector, OE: GES-1 cells stably expressing MAEL cDNA. (D) Adenovirus-mediated ILKAP overexpression suppresses MAEL-induced phosphorylation of ILKAP substrates in GES-1 cells. The adenovirus expressing GFP (Ad-GFP) or ILKAP (Ad-ILKAP) was infected into the uninfected GES-1 cells (Mock), GES-1 cells stably expressing empty vector (NC) or MAEL cDNA (OE). At $48 \mathrm{~h}$ post-infection, Western blotting was performed to detect phosphorylation of ILKAP substrates. Staining intensity was quantitated by Image J software. The number below the blots indicates relative band intensity of MAEL protein normalized against that of ACTB. 
that MAEL negatively regulated ILKAP protein levels by promoting its lysosome-dependent degradation in GC cells, while treatment with the proteasome inhibitor MG132 has no significant effect on the MAELmediated degradation of ILKAP protein (Figure 5F and Supplementary Figure 7). ILKAP is a member of the PP2C phosphatase family. It functions as a tumor suppressor $[27,29,30]$, is down-regulated in malignant melanoma [31] and is deleted in oral cancer [32]. Based on our findings, $M A E L$ overexpression contributes to the depression of ILKAP in gastric cancer. Moreover, rescuing ILKAP can counteract the influence of $M A E L$ overexpression on cell proliferation, colony formation, migration and invasion. These data suggest that $M A E L$ exerts oncogenic effects in an ILKAP-dependent way.

The first identified substrate of ILKAP was ILK1, which promotes anchorage-independence, motility, apoptosis and angiogenesis. ILK1 has been reported to mediate the phosphorylation of PKB on Ser473 and GSK3 $\beta$ on Ser9, while ILKAP selectively inhibits ILKmediated GSK3 $\beta$ phosphorylation, but does not affect AKT phosphorylation [25, 26]. Given that MAEL

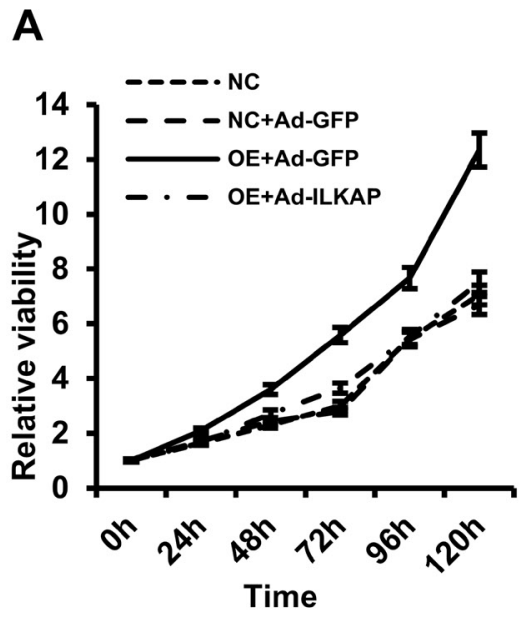

C
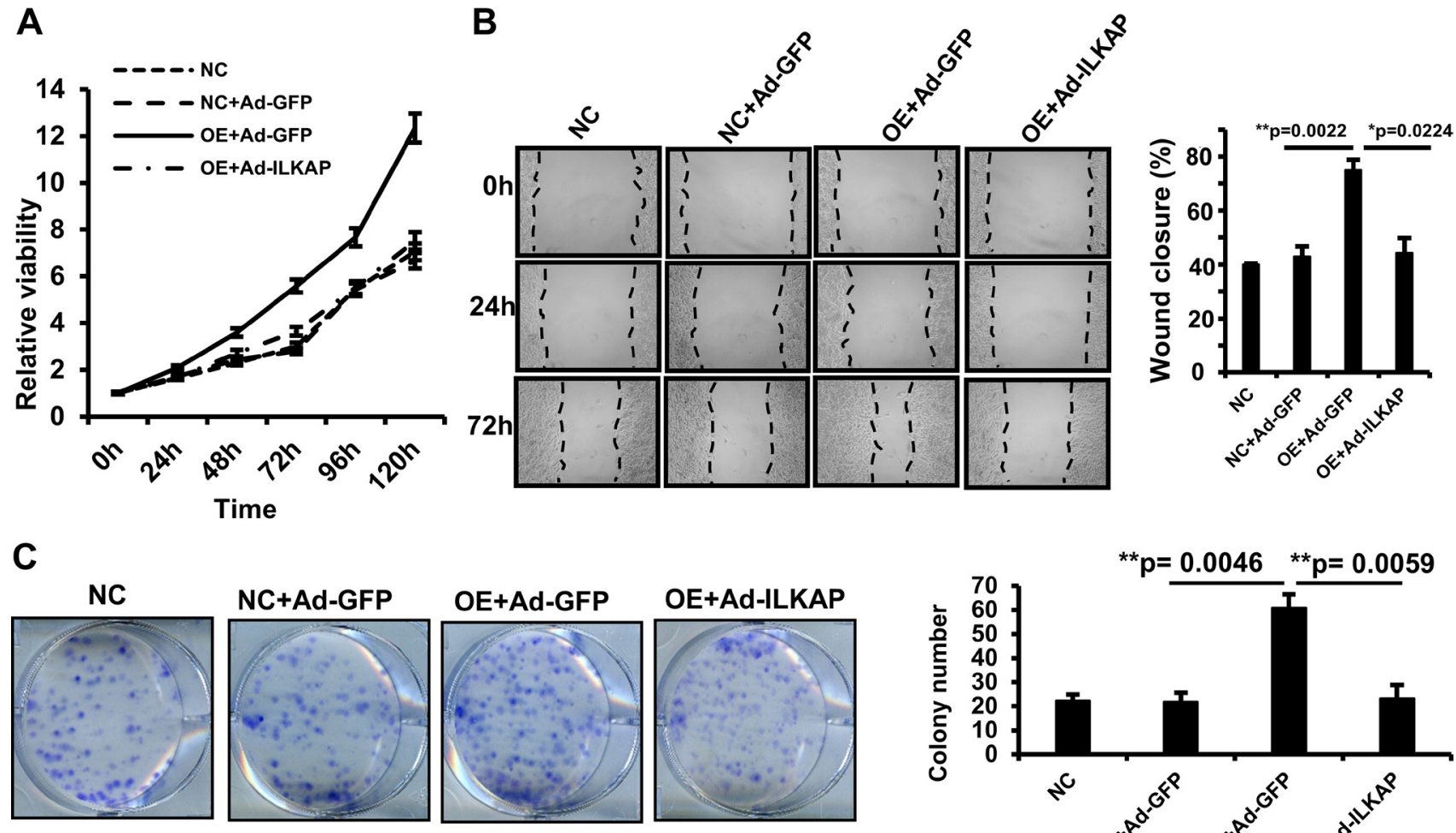

D
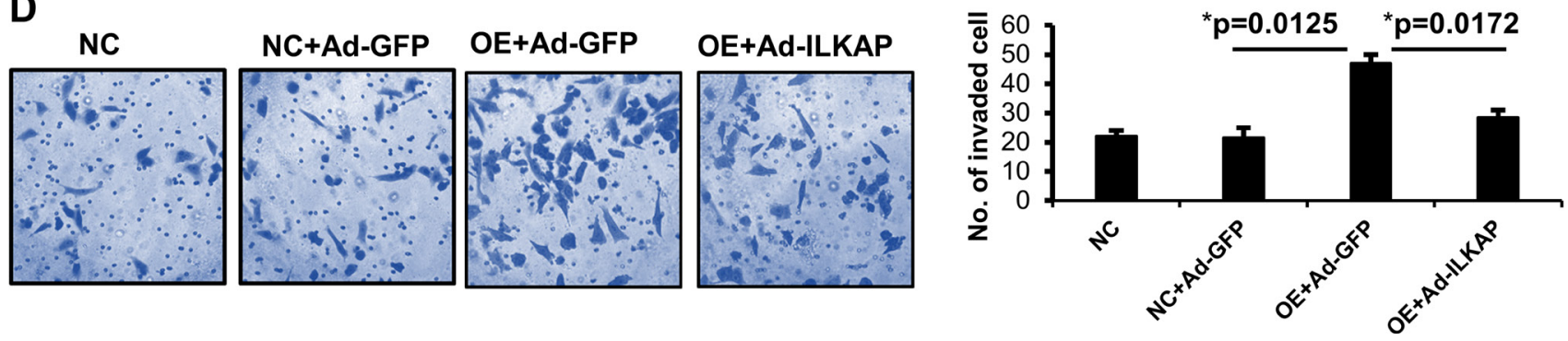

Figure 7: Adenovirus-mediated $I L K A P$ overexpression suppresses the oncogenic roles of $\boldsymbol{M A E L}$. The GES-1 cells stably expressing $M A E L$ were infected with adenovirus expressing GFP or ILKAP. (A) Dynamic changes of cell viability determined by MTT assay. The cells were harvested for MTT assay at different time after adenovirus infection. (B) Representative images (left panel) of wound area at the indicated time and percentage of wound closure at $72 \mathrm{~h}$ after scratching (right panel). (C) Representative images of colony (left panel) and the colony numbers (right panel). (D) Representative images (left panel) and statistical analysis (right panel) of invaded cells in the Transwell invasion assay. Mock: GES-1 cells, NC: GES-1 cells stably expressing empty vector; OE: GES-1 cells stably expressing MAEL cDNA, Ad-GFP: adenovirus expressing GFP, Ad-ILKAP: adenovirus expressing ILKAP. All the values were presented as means \pm S.D for at least three independent in vitro experiments. Differences between two groups were analyzed by student $t$-test; ${ }^{*} p$-value $\leq 0.05$ and ${ }^{* *} p$-value $\leq 0.01$. 
enhances ILKAP degradation, it should induce GSK3 $\beta$ phosphorylation. However, we did not observe any effects by MAEL on GSK3 $\beta$ or AKT phosphorylation. This result is consistent with a study in bladder cancer [13]. In contrast, Liu et al. demonstrated that MAEL increased AKT and GSK3 $\beta$ phosphorylation in hepatocellular carcinoma [11]. It is possible that $M A E L$ regulates different pathways in different cancer types. ILKAP has also been found to dephosphorylate RSK2, decrease the expression of its substrate cyclin D1, and then induce cell apoptosis [27]. Therefore, we investigated the effects of MAEL on RSK2 phosphorylation. As expected, MAEL knockdown in gastric cells increased ILKAP levels and decreased RSK2 phosphorylation levels, while MAEL overexpression decreased ILKAP protein levels and induced RSK2 phosphorylation. Moreover, adenovirus-mediated ILKAP overexpression significantly reversed MAEL-induced RSK2 phosphorylation. This suggests that MAEL suppresses the ILKAP-mediated dephosphorylation of RSK2. Kim et al. identified p38 and CHK1 as potential ILKAP substrates using an affinity pulldown assay [24]. However, it is unknown whether ILKAP dephosphorylates p38 and CHK1/2 in vivo. Here, we showed that MAEL increased p38 and CHK1 phosphorylation and that the MAEL-induced phosphorylation of p38 and CHK1 can be attenuated by ILKAP. RSK2, CHK1 and p38 have been reported to be associated with tumor growth, survival and chemotherapy resistance. Constitutive activation of p38 has been reported as a major causative factor of carcinogenesis and chemotherapy resistance in GC $[33,34]$. CHK1 ablation by small interfering RNAs significantly inhibits cell proliferation and sensitizes the effects of ionizing radiation (IR) treatment for GC [35]. Based on the above findings, MAEL exerts oncogenic effects by promoting ILKAP degradation and activating RSK2, CHK1 and p38.

In summary, we have demonstrated that $M A E L$ expression may serve as an unfavorable prognostic marker for GC patients and defined a novel oncogenic pathway for $M A E L$. Ectopic expression of MAEL stimulates the phosphorylation and activation of $\mathrm{p} 38, \mathrm{CHK} 1$ and RSK2 by inducing the degradation of the phosphatase ILKAP, which facilitates tumor growth and metastasis. Moreover, in a xenograft tumor mouse model, we showed that silencing $M A E L$ expression or intratumorally injecting adenoviruses expressing ILKAP suppresses tumor growth. Therefore, upregulating ILKAP expression and interfering with $M A E L$ expression may be useful gene therapy strategies for GC.

\section{MATERIALS AND METHODS}

\section{Online analysis}

The differential expression of $M A E L$ mRNA between cancerous and noncancerous tissues was analyzed using the microarray datasets in Oncomine (http:// www.oncomine.org), a cancer microarray database and integrated data-mining platform [36]. In this study, we used the NCBI GEO dataset GSE27342 for analysis of MAEL mRNA expression, which includes transcriptomic data of paired tumor and adjacent normal tissues from 80 gastric cancer patients [17]. Kaplan-Meier survival curves were calculated and plotted using the Kaplan-Meier Plotter online tool (http://kmplot.com) [21], which includes gene expression data and survival information of a total of 1,051 GC patients derived from NCBI GEO datasets GSE14210 ( $n=146)$, GSE15459 $(n=200)$, GSE22377 $(n=43), \operatorname{GSE} 29272(n=268), \operatorname{GSE} 51105(n=94)$, and GSE62254 $(n=300)$. All the above six datasets were used for exploring prognostic role of MAEL mRNA expression in GC. The cBioPortal [37] tool (http://www.cbioportal. org) was used to analyze the correlation between gene expression and copy number/methylation based on the cancer genomics data (Stomach Adenocarcinoma, TCGA, Provisional), which includes mRNA expression, DNA methylation, copy number variation, somatic mutation data of 478 gastric adenocarcinoma tissue cases.

\section{Cell lines, plasmids, antibodies and chemicals}

The human embryonic kidney cell line HEK293, gastric epithelial cell lines (GES-1 and RGM1) and GC cell lines (AGS, HGC-27, KATO-III, SUN-1, N87, and BGC-823) were purchased from ATCC (Manassas, VA, USA). The Myc-tagged MAEL expression construct MycMAEL was previously described [22]. The Myc-MAEL plasmid was used a template to construct the deletion mutants of $M A E L$ by overlapping PCR. The ILKAP coding sequence was cloned into the pCMV-HA vector at the SalI and KpnI sites, generating the HA-tagged ILKAP expression construct HA-ILKAP. The antibodies and chemicals used in this study are shown in Supplementary Tables 1 and 2 .

\section{Tissue specimens}

Gastric cancer and adjacent noncancerous tissues were collected from The First Affiliated Hospital, Hunan Normal University. The samples were histologically confirmed by staining with hematoxylin and eosin. The clinicopathological parameters of the gastric cancer patients were listed in Supplementary Table 3. This study was approved by the Ethics Committee of Hunan Normal University.

\section{Generation of stable cell lines}

The lentiviral particles expressing the $M A E L$ coding sequence or empty vector (GeneChem, Shanghai, China) were transduced into the gastric epithelial cell line GES-1, and puromycin was used for the selection and maintenance of the stable cells. The lentiviral 
particles expressing MAEL shRNA or scrambled shRNA (GeneCopoeia, Guangzhou, China) were used to infect the AGS and HGC-27 GC cell lines. The target sequence (GGAACTGGCCACCTATCTACT) for MAEL was described previously by Li et al. [13]. After infection, the cells were selected with hygromycin B.

\section{Methylation analysis by pyrosequencing}

The DNA methylation status of MAEL gene promoter was analyzed using pyrosequencing assay as described by Kim et al. [28]. Genomic DNA was processed with EpiTect Bisulfite conversion kit (Qiagen, Valencia, CA, USA) according to the manufacturer's instruction. The bisulfite-modified DNA was amplified using a forward primer and a biotinylated reverse primer. Amplification was performed in the Veriti 96-well thermal cycler (Qiagen) as follows: a pre-incubation at $95^{\circ} \mathrm{C}$ for $3 \mathrm{~min}$, followed by 50 cycles of $95^{\circ} \mathrm{C}$ for $15 \mathrm{sec}, 52^{\circ} \mathrm{C}$ for $20 \mathrm{sec}$ and $72^{\circ} \mathrm{C}$ for $30 \mathrm{sec}$, and a final extension at $72^{\circ} \mathrm{C}$ for $5 \mathrm{~min}$. Pyrosequencing reactions were carried out with a sequencing primer on the PyroMark Q96 system (Qiagen) according to the manufacturer's specifications. The primers for amplification and pyrosequencing were described previously by Kim et al. [28]. The sequence to be detected by pyrosequencing is CGCGGAAACACCCGC, which includes $3 \mathrm{CpG}$ dinucleotides (Supplementary Figure 2). An overall MAEL promoter methylation level was calculated as the average of the proportions of $\mathrm{C}$ (\%) at the $3 \mathrm{CpG}$ sites. SigmaPlot (Systat Software, San Jose, CA) was used for graphing and statistical analysis of correlation between mRNA level and methylation.

\section{RNA preparation, cDNA synthesis and real-time PCR}

Total RNA was extracted from cell lines or tissues using TRIzol reagent (Invitrogen, Carlsbad, CA, USA), and then reverse transcribed into cDNA using M-MLV RTase and a random primer (GeneCopoeia, Guangzhou, China). SYBR green-based realtime PCR was performed ABI 7900 thermocycler (Thermo Fisher Scientific, MA, USA). The primers were described previously [10].

\section{Adenovirus transduction}

The adenoviruses expressing ILKAP or GFP (Obio Technology Corp, Shanghai, China) were infected into cells at 50 multiplicity of infection (MOI).

\section{Xenograft tumor model}

BALB/c nude mice (7-8 weeks old) were purchased from SLACCAS Jingda (Changsha, China). A total of $200 \mu \mathrm{L}$ of serum-free DMEM containing $5 \times 10^{6}$ cells (HGC-27 cells stably expressing the scrambled or MAEL shRNAs) or $1 \times 10^{7}$ cells (GES- 1 cells stably expressing the empty vector or $M A E L$ ) was injected into the back of nude mice. The length and width of the tumors were measured by a vernier caliper every 3 days, and the tumor volume was calculated by the following formula: volume $=$ width $^{2} \times$ length $/ 2$. The experiments were approved by the Animal Care and Use Committee of Hunan Normal University, and all animals were handled in accordance with the guidelines of the Hunan Provincial Council on Animal Care.

\section{Co- IP and western blotting}

Co-IP and Western blotting were performed as described previously [38]. Briefly, HEK293 cells were co-transfected with HA-ILKAP plasmid and Myc-tagged wildtype or deletion mutants of $M A E L$ and harvested at $24 \mathrm{~h}$ post-transfection. Cell lysates were prepared and immunoprecipitated with rabbit anti-HA/Myc antibody or pre-immune rabbit $\mathrm{IgG}$, and the precipitated proteins were detected by Western blot analysis using murine antiMyc and anti-HA antibodies. For co-IP of endogenous ILKAP and MAEL proteins, lysates of AGS cells were immunoprecipitated with mouse anti-ILKAP antibody or pre-immune rabbit IgG, and the precipitated proteins were detected by Western blot analysis using rabbit anti-MAEL and mouse anti-ILKAP antibodies.

\section{MTT cell proliferation}

The cells were cultured in serum-free medium for $24 \mathrm{~h}$ and then seeded in 96-well plates with normal medium for the indicated times. Cell viability was determined by the MTT assay as described previously [39].

\section{Wound-healing and invasion assays}

For wound-healing assays, cells were cultured in a 24-well plate until they reached $90 \%$ confluency and were subsequently serum-starved overnight. After scratching, the cells were cultured in medium with a low concentration of serum (1\%) until complete closure of the scratch wound was observed. Photos were obtained at different time points using an inverted microscope. Invasion assays were performed using a Corning Matrigel invasion chamber (Tewksbury, MA, USA), which consists of a cell culture insert with an 8 - $\mu \mathrm{m}$ pore size PET membrane uniformly coated with Matrigel matrix.

\section{Colony formation assay}

Cells were cultured in 6-well plates until colonies became visible to the naked eye. The colonies were subsequently rinsed with PBS, fixed with methanol and stained with Giemsa. 


\section{Statistical analysis}

All the statistical analyses were performed with Excel 2010 (Microsoft, Seattle, WA, USA). The values are presented as the mean \pm S.D. Differences between two groups were analyzed with Student's $t$-test. The level of statistical significance is expressed as the $p$-value; ${ }^{*} p$-value $\leq 0.05$ and $^{* *} p$-value $\leq 0.01$.

\section{ACKNOWLEDGMENTS AND FUNDING}

This work was supported by the National Natural Science Foundation of China (grant no. 81272318), the Cooperative Innovation Center of Engineering and New Products for Developmental Biology of Hunan Province (grant no. 20134486), the Health and Family Planning Commission of Hunan Province (grant no. B2014-090) and the Hunan Provincial Science and Technology Department (grant no. 2015SK2044). The results published here are in part based upon data from GEO, Oncomine and TCGA.

\section{CONFLICTS OF INTEREST}

The authors declare no conflicts of interests.

\section{REFERENCES}

1. Torre LA, Bray F, Siegel RL, Ferlay J, Lortet-Tieulent J, Jemal A. Global cancer statistics, 2012. CA Cancer J Clin. 2015; 65:87-108.

2. Ahmad SA, Xia BT, Bailey CE, Abbott DE, Helmink BA, Daly MC, Thota R, Schlegal C, Winer LK, Ahmad SA, Al Humaidi AH, Parikh AA. An update on gastric cancer. Curr Probl Surg. 2016; 53:449-490.

3. Rocken C. Molecular classification of gastric cancer. Expert Rev Mol Diagn. 2017; 17:293-301.

4. Feichtinger J, Larcombe L, McFarlane RJ. Meta-analysis of expression of 1(3)mbt tumor-associated germline genes supports the model that a soma-to-germline transition is a hallmark of human cancers. Int J Cancer. 2014; 134:2359-2365.

5. Van Tongelen A, Loriot A, De Smet C. Oncogenic roles of DNA hypomethylation through the activation of cancergermline genes. Cancer Lett. 2017; 396:130-137.

6. Janic A, Mendizabal L, Llamazares S, Rossell D, Gonzalez C. Ectopic expression of germline genes drives malignant brain tumor growth in Drosophila. Science. 2010; 330:1824-1827.

7. Clegg NJ, Frost DM, Larkin MK, Subrahmanyan L, Bryant Z, Ruohola-Baker H. maelstrom is required for an early step in the establishment of Drosophila oocyte polarity: posterior localization of grk mRNA. Development. 1997; 124:4661-4671.

8. Soper SF, van der Heijden GW, Hardiman TC, Goodheart M, Martin SL, de Boer P, Bortvin A. Mouse maelstrom, a component of nuage, is essential for spermatogenesis and transposon repression in meiosis. Dev Cell. 2008; 15:285-297.

9. Costa Y, Speed RM, Gautier P, Semple CA, Maratou K, Turner JM, Cooke HJ. Mouse MAELSTROM: the link between meiotic silencing of unsynapsed chromatin and microRNA pathway? Hum Mol Genet. 2006; 15:2324-2334.

10. Xiao L, Wang Y, Zhou Y, Sun Y, Sun W, Wang L, Zhou C, Zhou J, Zhang J. Identification of a novel human cancer/ testis gene MAEL that is regulated by DNA methylation. Mol Biol Rep. 2010; 37:2355-2360.

11. Liu L, Dai Y, Chen J, Zeng T, Li Y, Chen L, Zhu YH, Li J, Li Y, Ma S, Xie D, Yuan YF, Guan XY. Maelstrom promotes hepatocellular carcinoma metastasis by inducing epithelialmesenchymal transition by way of Akt/GSK-3beta/Snail signaling. Hepatology. 2014; 59:531-543.

12. Li Q, Wei P, Huang B, Xu Y, Li X, Li Y, Cai S, Li D. MAEL expression links epithelial-mesenchymal transition and stem cell properties in colorectal cancer. Int J Cancer. 2016; 139:2502-2511.

13. Li XD, Zhang JX, Jiang LJ, Wang FW, Liu LL, Liao YJ, Jin XH, Chen WH, Chen X, Guo SJ, Zhou FJ, Zeng YX, Guan XY, et al. Overexpression of maelstrom promotes bladder urothelial carcinoma cell aggressiveness by epigenetically downregulating MTSS1 through DNMT3B. Oncogene. 2016; 35:6281-6292.

14. Kim SH, Park ER, Cho E, Jung WH, Jeon JY, Joo HY, Lee KH, Shin HJ. Mael is essential for cancer cell survival and tumorigenesis through protection of genetic integrity. Oncotarget. 2017; 8:5026-5037. https://doi.org/10.18632/ oncotarget. 13756.

15. Lim SL, Ricciardelli C, Oehler MK, Tan IM, Russell D, Grutzner F. Overexpression of piRNA pathway genes in epithelial ovarian cancer. PLoS One. 2014; 9:e99687.

16. Benstead-Hume G, Wooller SK, Pearl FMG. 'Big data' approaches for novel anti-cancer drug discovery. Expert Opin Drug Discov. 2017; 12:599-609.

17. Cui J, Chen Y, Chou WC, Sun L, Chen L, Suo J, Ni Z, Zhang M, Kong X, Hoffman LL, Kang J, Su Y, Olman V, et al. An integrated transcriptomic and computational analysis for biomarker identification in gastric cancer. Nucleic Acids Res. 2011; 39:1197-1207.

18. Lee J, Kotliarova S, Kotliarov Y, Li A, Su Q, Donin NM, Pastorino S, Purow BW, Christopher N, Zhang W, Park JK, Fine HA. Tumor stem cells derived from glioblastomas cultured in bFGF and EGF more closely mirror the phenotype and genotype of primary tumors than do serumcultured cell lines. Cancer Cell. 2006; 9:391-403.

19. Finak G, Bertos N, Pepin F, Sadekova S, Souleimanova M, Zhao H, Chen H, Omeroglu G, Meterissian S, Omeroglu A, Hallett M, Park M. Stromal gene expression predicts clinical outcome in breast cancer. Nat Med. 2008; 14:518-527.

20. Okayama H, Kohno T, Ishii Y, Shimada Y, Shiraishi K, Iwakawa R, Furuta K, Tsuta K, Shibata T, Yamamoto S, 
Watanabe S, Sakamoto H, Kumamoto K, et al. Identification of genes upregulated in ALK-positive and EGFR/KRAS/ ALK-negative lung adenocarcinomas. Cancer Res. 2012; 72:100-111.

21. Szasz AM, Lanczky A, Nagy A, Forster S, Hark K, Green JE, Boussioutas A, Busuttil R, Szabo A, Gyorffy B. Crossvalidation of survival associated biomarkers in gastric cancer using transcriptomic data of 1,065 patients. Oncotarget. 2016; 7:49322-49333. https://doi.org/10.18632/ oncotarget.10337.

22. Yuan L, Xiao Y, Zhou Q, Yuan D, Wu B, Chen G, Zhou J. Proteomic analysis reveals that MAEL, a component of nuage, interacts with stress granule proteins in cancer cells. Oncol Rep. 2014; 31:342-350.

23. Tong Y, Quirion R, Shen SH. Cloning and characterization of a novel mammalian PP2C isozyme. J Biol Chem. 1998; 273:35282-35290.

24. Hojlys-Larsen KB, Sorensen KK, Jensen KJ, Gammeltoft S. Probing protein phosphatase substrate binding: affinity pulldown of ILKAP phosphatase $2 \mathrm{C}$ with phosphopeptides. Mol Biosyst. 2012; 8:1452-1460.

25. Leung-Hagesteijn C, Mahendra A, Naruszewicz I, Hannigan GE. Modulation of integrin signal transduction by ILKAP, a protein phosphatase $2 \mathrm{C}$ associating with the integrin-linked kinase, ILK1. EMBO J. 2001; 20:2160-2170.

26. Kumar AS, Naruszewicz I, Wang P, Leung-Hagesteijn C, Hannigan GE. ILKAP regulates ILK signaling and inhibits anchorage-independent growth. Oncogene. 2004; 23:3454-3461.

27. Zhou W, Cao H, Yang X, Cong K, Wang W, Chen T, Yin H, Wu Z, Cai X, Liu T, Xiao J. Characterization of nuclear localization signal in the $\mathrm{N}$ terminus of integrin-linked kinase-associated phosphatase (ILKAP) and its essential role in the down-regulation of RSK2 protein signaling. J Biol Chem. 2013; 288:6259-6271.

28. Kim YH, Lee HC, Kim SY, Yeom YI, Ryu KJ, Min BH, Kim DH, Son HJ, Rhee PL, Kim JJ, Rhee JC, Kim HC, Chun HK, et al. Epigenomic analysis of aberrantly methylated genes in colorectal cancer identifies genes commonly affected by epigenetic alterations. Ann Surg Oncol. 2011; 18:2338-2347.

29. Hausmann C, Temme A, Cordes N, Eke I. ILKAP, ILK and PINCH1 control cell survival of p53-wildtype glioblastoma cells after irradiation. Oncotarget. 2015; 6:34592-34605. https://doi.org/10.18632/oncotarget.5423.

30. Lorenzato A, Torchiaro E, Olivero M, Di Renzo MF. The integrin-linked kinase-associated phosphatase (ILKAP) is a regulatory hub of ovarian cancer cell susceptibility to platinum drugs. Eur J Cancer. 2016; 60:59-68.

31. Arroyo-Berdugo Y, Alonso S, Ribas G, Ibarrola-Villava M, Pena-Chilet M, Martinez-Cadenas C, Gardeazabal J, RatonNieto JA, Sanchez-Diez A, Careaga JM, Perez-Yarza G, Carretero G, Martin-Gonzalez M, et al. Involvement of ANXA5 and ILKAP in susceptibility to malignant melanoma. PLoS One. 2014; 9:e95522.

32. Cengiz B, Gunduz M, Nagatsuka H, Beder L, Gunduz E, Tamamura R, Mahmut N, Fukushima K, Ali MA, Naomoto Y, Shimizu K, Nagai N. Fine deletion mapping of chromosome 2q21-37 shows three preferentially deleted regions in oral cancer. Oral Oncol. 2007; 43:241-247.

33. Guo X, Ma N, Wang J, Song J, Bu X, Cheng Y, Sun K, Xiong H, Jiang G, Zhang B, Wu M, Wei L. Increased p38MAPK is responsible for chemotherapy resistance in human gastric cancer cells. BMC Cancer. 2008; 8:375.

34. Graziosi L, Mencarelli A, Santorelli C, Renga B, Cipriani S, Cavazzoni E, Palladino G, Laufer S, Burnet M, Donini A, Fiorucci S. Mechanistic role of p38 MAPK in gastric cancer dissemination in a rodent model peritoneal metastasis. Eur J Pharmacol. 2012; 674:143-152.

35. Yin Y, Shen Q, Zhang P, Tao R, Chang W, Li R, Xie G, Liu W, Zhang L, Kapoor P, Song S, Ajani J, Mills GB, et al. Chk1 inhibition potentiates the therapeutic efficacy of PARP inhibitor BMN673 in gastric cancer. Am J Cancer Res. 2017; 7:473-483.

36. Rhodes DR, Kalyana-Sundaram S, Mahavisno V, Varambally R, Yu J, Briggs BB, Barrette TR, Anstet MJ, Kincead-Beal C, Kulkarni P, Varambally S, Ghosh D, Chinnaiyan AM. Oncomine 3.0: genes, pathways, and networks in a collection of 18,000 cancer gene expression profiles. Neoplasia. 2007; 9:166-180.

37. Gao J, Aksoy BA, Dogrusoz U, Dresdner G, Gross B, Sumer SO, Sun Y, Jacobsen A, Sinha R, Larsson E, Cerami E, Sander C, Schultz N. Integrative analysis of complex cancer genomics and clinical profiles using the cBioPortal. Sci Signal. 2013; 6:pl1.

38. Zhou J, Qiao X, Xiao L, Sun W, Wang L, Li H, Wu Y, Ding $\mathrm{X}, \mathrm{Hu} \mathrm{X}$, Zhou $\mathrm{C}$, Zhang J. Identification and characterization of the novel protein CCDC106 that interacts with p53 and promotes its degradation. FEBS Lett. 2010; 584:1085-1090.

39. Zhou J, Duan H, Xie Y, Ning Y, Zhang X, Hui N, Wang C, Zhang J, Zhou J. MiR-193a-5p Targets the Coding Region of AP-2alpha mRNA and Induces Cisplatin Resistance in Bladder Cancers. J Cancer. 2016; 7:1740-1746. 